\title{
Do Women with Diabetes Need More Intensive Action for Cardiovascular Reduction than Men with Diabetes?
}

\author{
Jürgen Harreiter ${ }^{1}$ (D) - Helena FadI ${ }^{2}$ (D) - Alexandra Kautzky-Willer ${ }^{1,3}$ (D) David Simmons ${ }^{2,4}$ (D)
}

Accepted: 10 September 2020 / Published online: 9 October 2020

(C) The Author(s) 2020

\begin{abstract}
Purpose of Review This narrative review makes the case for greater efforts to reduce cardiovascular disease (CVD) risk in women with diabetes.

Recent Findings In a recent meta-analysis including five CVOTs of diabetes medications with 46,606 subjects, women (vs men) with type 2 diabetes had a higher relative risk for stroke (RR 1.28; 95\% CI 1.09, 1.50) and heart failure (1.30; 1.21, 1.40). Prior studies found higher "within-gender" RR for CVD mortality in women with diabetes although men have an absolute higher risk. Women with prior gestational diabetes mellitus (GDM) have a 2-fold higher CVD risk than the background population. Worse CVD and CVD risk factor management in women, as well as lower female therapy adherence, contribute further to these disparities.

Summary The mechanism behind this excess risk includes biological, hormonal, socioeconomic, clinical, and behavioral factors that still require further investigation. The need for more intensive CVD reduction in women now includes more attention to screening for both incident diabetes and CVD risk factors among high-risk women.
\end{abstract}

Keywords Diabetes mellitus $\cdot$ Gestational diabetes $\cdot$ Cardiovascular disease $\cdot$ Sex $\cdot$ Gender $\cdot$ Prevention $\cdot$ Diabetes management $\cdot$ CVD risk factors $\cdot$ Lipids $\cdot$ Sex hormones

This article is part of the Topical Collection on Macrovascular Complications in Diabetes

Jürgen Harreiter

juergen.harreiter@meduniwien.ac.at

Helena Fadl

helena.fad1@oru.se

Alexandra Kautzky-Willer

alexandra.kautzky-willer@meduniwien.ac.at

David Simmons

da.simmons@westernsydney.edu.au

1 Gender Medicine Unit, Division of Endocrinology and Metabolism, Department of Medicine III, Medical University of Vienna, Spitalgasse 23, 1090 Vienna, Austria

2 Department of Obstetrics and Gynaecology, Faculty of Medicine and Health, Örebro University, SE 70182 Örebro, Sweden

3 Gender Institute, Gars am Kamp, Austria

4 Macarthur Clinical School, Western Sydney University, Sydney, New South Wales, Australia

\section{Introduction}

In the general population, women die at an older age than men in nearly all parts of the world such that life expectancy is on average 4 years higher among women than men [1]. These differences have been attributed to biological, socioeconomic, environmental, and behavioral factors [2-4]. Although women live longer, this advantage frequently fails to translate into increased healthy life years [5]. Cardiovascular disease (CVD) is the leading cause of death among both men and women [6]. Diabetes is a wellknown CVD risk factor and thus patients with diabetes mellitus have a high need for approaches that will reduce premature mortality from CVD. The number of people with diabetes worldwide is predicted to increase from the current 463 million to 700 million by 2045 [7], and with it, the number of people developing CVD associated with diabetes. While there are only small gender differences in the age-specific prevalence of diabetes in adulthood [8••], there are sex and gender differences in the relationship between diabetes and CVD mortality. 
Since the Framingham study is nearly five decades ago, it has been clear that the usual "female protection" is lost among women with insulin-treated diabetes, and that this is based on their higher CVD risk [9]. Several other studies subsequently demonstrated the increased relative risk for CVD in women with diabetes compared with men, in both type 1 (T1DM) and type $2(\mathrm{~T} 2 \mathrm{DM})$ diabetes $[10 \bullet, 11,12 \bullet, 13 \bullet \bullet]$. An excess CVD risk has also been shown among women with prior gestational diabetes mellitus (GDM) [14••]. The exact pathophysiological explanations remain under-investigated and need further research, but the higher risk seems to be driven by biological, environmental, and behavioral factors [8••]. Biological causes discussed include a loss of the protective hormonal effect by female sex hormones and sex hormone imbalance in hyperglycemic conditions leading to higher oxidative stress and endothelial dysfunction, a proinflammatory environment acting on estrogen receptor actions, a modulation of vascular response to nitric oxide, and impaired vessel relaxation properties [8••]. Further proposed explanations for the excess CVD mortality reported in women with diabetes compared with those without diabetes include pregnancy-related conditions and sex and gender differences in diabetes management and the management of other CVD risk factors [15] including hypertension [16].

In view of this sex and gender difference in excess CVD risk, our narrative review poses the question, whether all or, at least some women with diabetes, are in need of increased and more aggressive cardiovascular risk reduction than men. This includes the need for any further advances in diabetes prevention, treatment, CVD risk reduction, or management. We further attempt to shed light on the biological and socio-cultural aspects that are potentially responsible for these sex- and gender-related disparities.

\section{Why Do Women Live longer?}

In general, in high-income populations, life expectancy is 47 years higher in women than in men [17], whereas in lowincome countries, a smaller gap was reported as women have less access to health care services [1]. Between the poorest and richest countries, the life expectancy gap opens up to more than 18 years as reported in a $2018 \mathrm{WHO}$ report [1]. There are some data to suggest that this survival difference is mainly due to modifiable risk factors with biological causes only playing a minor role. Key evidence comes from a study comparing 11,000 German male and female cloister inhabitants from Catholic communities with the general population. A maximum survival disadvantage of about 1 year of life expectancy from a young adult age ( 25 years) was found in the male cloister inhabitants compared with women from the cloister communities and the general population [18]. Men from the general population had a lower life expectancy from a young adult age compared with the cloister inhabitants. It was also proposed that these males were unable to decrease their mortality risk, in contrast to the female general population, nuns, and monks [18]. The protected environment of the cloister seemed to have positive effects on life expectancy on male inhabitants. Modifiable risk factors, such as smoking, alcohol, unhealthy food choices, excess body weight, and lack of physical inactivity, were more prevalent in the male general population [18].

While women have longer life expectancy, there is evidence to suggest that the balance between healthy and unhealthy live years includes a sex-specific imbalance, the socalled health survival paradox [5]. While women and men spend comparable time in a "good health" state, the longer life expectancy in women results in women accumulating more unhealthy life years compared with men.

\section{Increased Mortality Risk in Women with Diabetes?}

In general, the absolute risk for T2DM and CVD mortality is higher in men; however, the relative risk of all-cause and CVD mortality in women with T2DM has consistently been reported to be significantly higher and was also reported in women with T1DM (Table 1) $[12 \bullet, 19,20$, $21 \bullet, 22,23]$. Higher risks were observed in younger groups with diabetes compared with older groups with the highest risk found in women with diabetes between 35 and 59 years of age [22]. Some studies observed higher CVD risk in both sexes, with a non-significant higher relative risk in women [23]. Key findings pertinent to sex differences in CVD outcomes were the reported higher obesity, hypertension, and dyslipidemia, as well as lower prescription of medication among women [23]. Furthermore, the risk of heart failure was reportedly higher in women with T1DM and T2DM (Table 1) [24•, 25•]. There was a narrowing sex ratio within older age categories, indicating that relative risk is higher in women of younger age [24•].

Other studies also hypothesize a reduction in sex-related protection in women with diabetes, which is, in turn, associated with higher mortality risk $[8 \cdot \bullet, 27,28]$. Potential risk factors explaining these differences are shown in Fig. 1. In view of this substantial effect of diabetes on CVD risk, there have been discussions over the need for more intensive CVD reduction activities, and individualization of risk reduction approaches $[8 \bullet \bullet, 29,30 \bullet]$. It is obvious from the presented data that an individualized risk reduction approach needs to take into account both sex and age (e.g., the use of CVD risk reduction agents in women of reproductive age), but there also needs to be a focus on modifiable risk factors. 
Table 1 Studies reporting sex and gender differences in the effect of diabetes mellitus on all cause and cardiovascular disease mortality and heart failure

\begin{tabular}{lr} 
Author & Population \\
\hline Cardiovascular mortality & \\
Xu et al. [19] & $2,314,292$ individuals, among whom \\
254,038 all-cause deaths occurred
\end{tabular}

Wang et al. [20]

$5,162,654$ participants

Huxley et al. [21•] 447,064 participants

Huxley et al. [12•]

Prospective Studies Collaboration and Asia Pacific Cohort Studies

Collaboration [22]

980,793 participants and 76,965 fatalities different ethnicities and age categories, age $35-89$ years
Design

Systematic review and meta-analysis including prospective cohort 35 studies

Systematic review and meta-analysis including 49 studies

Meta-analysis of 37 prospective cohort studies

Meta-analysis of 26 studies
Meta-analysis of 68 prospective studies

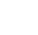

Outcome

Pooled women vs. men ratio of the HRs 1.17 (95\% CI: $1.12-1.23)$ and 1.97 (1.49-2.61) respectively for all cause and coronary heart disease (CHD) mortality.

increased all-cause mortality in men (HR 1.91 (1.72-2.12)) and women (2.33 (2.02-2.69)) with T2DM vs. healthy population

Higher relative all-cause mortality (RRR $1.13,95 \%$ CI $1.07-1.19 ; p<0.001)$ and CVD mortality $(1.30,1.13-1.49$; $p<0.001)$ in women with diabetes vs. men with diabetes.

Women with diabetes vs. men with diabetes: CHD mortality RRR 1.58 , 95\% CI 1.32-1.90; $p<0.001$ and stroke mortality $1.08,1.01-1.15$; $p<0.001$

Higher risk of fatal CHD in women with diabetes vs. men, pooled ratio of the RR 1.46 (1.14-1.88).

Fatal CHD in patients with diabetes vs. no diabetes significantly higher in women than men: $3.50(2.70-4.53)$ vs. 2.06 (1.81-2.34).

pooled women-to-men ratio in patients with T1DM: SMR 1.37 (95\% CI 1.21-1.56) for all-cause mortality, 1.44 (1.02-2.05) increased risk for renal disease mortality, 1.37 (1.03-1.81) for stroke mortality, CVD mortality 1.86 (1.62-2.15), 2.54 (1.80-3.60) for incident coronary heart disease

Doubling of occlusive vascular mortality risk in men with diabetes RR 2.10, 95\% CI 1.97-2.24), tripling in women with diabetes $(3.00,2.71-3.33)$ after stratification for age, total cholesterol, blood pressure, smoking status and BMI.

Higher risks in younger groups with diabetes aged $35-59$ years $(2.60$, 2.30-2.94) vs. older groups aged $70-89$ years $(2.01,1.85-2.19)$, highest risk in women with diabetes and age 35 and 59 years $(5.55$, 4.15-7.44). in absolute numbers, adjusted diabetes associated excess occlusive vascular mortality comparable in men and women across all age categories

Higher CVD event risk in both men and women, with a non-significant higher relative risk in women (RR 1.07 (0.98-1.17)

Higher risk for women for stroke (RR $1.28 ; 1.09-1.50)$, heart failure (1.30;
Clemens et al. [13••] 46,606 participants of trials examining the effect of diabetes medications on
Retrospective cohort study

Meta-analysis of 5 CVOTs on 3- or 4-point MACE (i.e., CV death, 
Table 1 (continued)

\begin{tabular}{|c|c|c|c|}
\hline Author & Population & Design & Outcome \\
\hline & $\begin{array}{l}\text { major adverse cardiovascular events } \\
\text { in people } \geq 18 \text { years of age with } \\
\text { T2DM }\end{array}$ & $\begin{array}{l}\text { nonfatal myocardial infarction, } \\
\text { nonfatal stroke, hospitalization for } \\
\text { heart failure, hospitalization for } \\
\text { unstable angina for 4-point MACE) }\end{array}$ & $\begin{array}{l}\text { 1.21-1.40), and CKD }(1.33 \text {; } \\
1.17-1.51) \text {, similar risk for PAD } \\
(1.12 ; 0.97-1.30) \text { and lower risk for } \\
\text { myocardial infarction }(0.71 \text {; } \\
0.59-0.86) \text {, consistently fewer female } \\
\text { participants }(28.5-35.8 \%) \text { in the trials. }\end{array}$ \\
\hline \multicolumn{4}{|l|}{ Heart failure } \\
\hline Malmborg et al. [24•] & $\begin{array}{l}218,549 \text { ( } 46 \% \text { women) participants, age } \\
40-89 \text { years }\end{array}$ & Population based study & $\begin{array}{l}\text { Higher absolute risk of MACE-HF in } \\
\text { men, but in relative terms } 15 \% \text { higher } \\
\text { women to men ratio }(95 \% \mathrm{Cl} \\
1.11-1.19, p<0.001) \text { at the age of } \\
50-60 \text { years. The risk of recurrent } \\
\text { CVD events or HF within } 30 \text { days } \\
\text { irrespective of diabetes or sex, more } \\
\text { often with increasing age. }\end{array}$ \\
\hline Ohkuma et al. [25•]. & $\begin{array}{l}12,142,998 \text { individuals and } 253,260 \\
\text { heart failure events }\end{array}$ & $\begin{array}{l}\text { Systematic review and meta-analysis of } \\
47 \text { cohorts }\end{array}$ & $\begin{array}{l}\text { Women with diabetes have increased } \\
\text { relative risk of heart failure vs. men: } \\
\text { T1DM (RRR } 1.47(1.44,1.90)) \text {, T2DM } \\
\quad(\text { RRR } 1.09(1.05,1.13))\end{array}$ \\
\hline Kannel et al. [26]. & 5209 men and women, age 30-62 years, & Framingham study, 18 year follow up & $\begin{array}{l}\text { Five- and two-fold increased risk of heart } \\
\text { failure in women and men with } \\
\text { diabetes respectively vs. healthy } \\
\text { population }\end{array}$ \\
\hline
\end{tabular}

$H R$, hazard ratios; $R R R$, relative risk reduction, defined as the relative decrease in the risk of an event in a cohort exposed compared with a cohort unexposed to a disease/risk; $S M R$, standardized mortality ratio, defined as an age and sex matched comparison of mortality in a cohort with a specific illness to controls; $R R$, relative risk, defined as the ratio of the likelihood of an event in an exposed compared to an unexposed cohort; $C V O T$, cardiovascular outcome trial

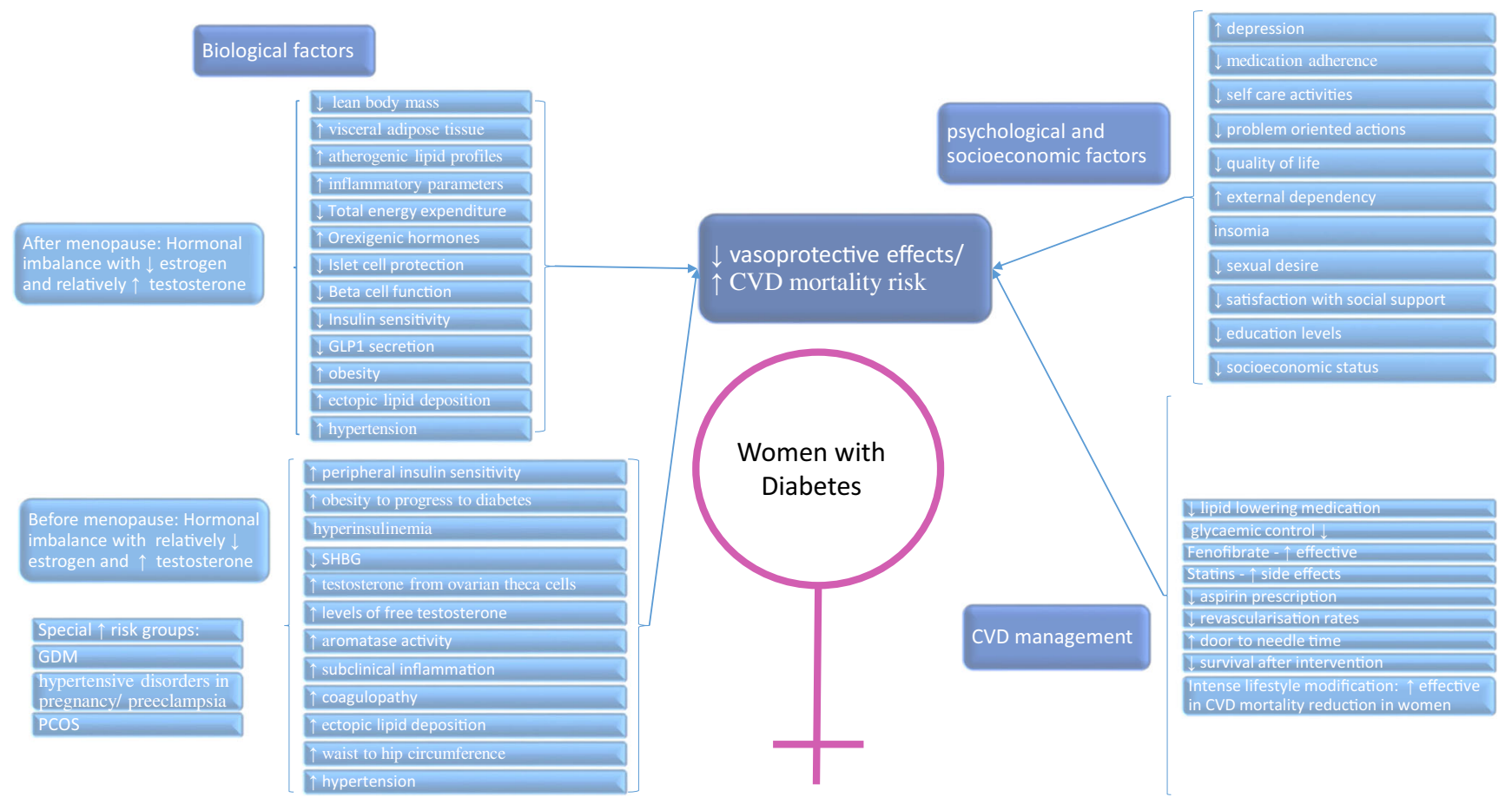

Fig. 1 Specific risk factors for progression to CVD and CVD mortality in women with diabetes mellitus 


\section{Are the Differences in Excess CVD Mortality Due to Sex Hormone Differences?}

Changes in hormonal action might contribute to the faster progression of CVD in both men and women with diabetes as a result of the effects on CVD risk factors as insulin resistance, overweight and obesity, and subsequently hyperglycemia, hypertension, and hypercholesterolemia evolve [31•]. The loss of female protection is therefore potentially driven by hormonal imbalance with the increased atherogenic lipid profiles, higher inflammation, or stronger relation of other CVD risk factors with hyperglycemia in women than men $[8 \bullet \cdot, 28]$. The contribution to the excess CVD among women with diabetes by pregnancy complications such as gestational hypertension and preeclampsia are also not fully understood, but early-onset preeclampsia has been shown to be associated with risk factors for CVD by the fifth decade of life [32].

Menopause is a particularly important time for CVD risk factors, when female sex hormone levels (e.g., estrogen) suddenly drop associated with a relative increase in male hormones (e.g., testosterone). This change is associated with weight gain, impairments in glucose metabolism and lipid storage, and finally progression to T2DM [8••]. After menopause, lean body mass declines and the prevalence of obesity increases $[33 \bullet \bullet, 34]$. The low estrogen levels after menopause reduce total energy expenditure through effects on the hypothalamus and higher release of orexigenic hormones [34]. Higher estrogen levels are also related to increased beta cell function and improved glycemic control in women with T2DM, and, additionally, slightly lower T2DM risk was reported in studies administering hormone replacement therapy in post-/menopausal healthy women $[35 \bullet \cdot$. Between the ages of 50 and 59 years, and within 10 years after menopause onset, hormone replacement therapy is effective in preventing the development of T2DM and cardiovascular disease in women without diabetes, although safety concerns persist with the need to balance benefits with risks [35••]. Endogenous estrogen in women might exert vasoprotective effects and thus lower cardiovascular events in women-a benefit which seems attenuated in women with hyperglycemia $[8 \cdot \bullet, 31 \bullet]$.

Female sex hormones and especially endogenous estrogen seem to have beneficial and protective effects on islet cells by preventing islet cell apoptosis driven by oxidative stress and lipotoxicity [36]. Higher insulin production and secretion were found along with approximately $6 \%$ higher beta cell content in pancreatic biopsies of women compared with men. Higher insulin secretion in women was associated with sex differences in glucagon-like peptide-1 (GLP-1) secretion, which was found to be $20 \%$ higher in normo-glucose-tolerant women compared with healthy men [36]. These differences found between women and men did not exist after progression to prediabetes or diabetes, independent of weight or age suggesting loss of protective effect with progression to diabetes [36].

An ambivalent role has been described for testosterone. High testosterone levels in women are associated with insulin resistance, hyperglycemia, and central obesity as well as hypertension $[2,35 \bullet \bullet]$. Paradoxically, low testosterone levels in men seem to have similar effects on CVD risk factors demonstrating the duality of the mechanism of this hormone $[8 \cdot \bullet$, $37 \bullet \bullet]$. These considerations are supported by studies providing evidence of adverse cardiometabolic consequences in women with PCOS, a state of relative testosterone excess in women, such as atherogenic lipid profiles, inflammation, fatty liver disease, hypertension, diabetes, and coagulopathy [38]. Indeed, in women with obesity, sex hormone-binding globulin (SHBG) levels are lower-associated with hyperinsulinemia and increased liver fat - a state related with higher diabetes risk in women - and further contributing to higher levels of free testosterone [8••]. Higher insulin levels resulting from higher insulin resistance contribute to an increase in insulin-mediated ovarian testosterone production in thecal cells, further contributing to sex hormone imbalance $[39,40]$.

\section{Modifiable CVD Risk Factors: Evidence for the Need for Additional and More Intensive Risk Reduction Strategies Among Women}

Evidence for the need for additional and more intensive risk reduction strategies among women can be found in the study of the Prospective Studies Collaboration and Asia Pacific Studies Collaboration, where excess mortality risk among women with diabetes was investigated within other modifiable cardiovascular risk factors as BMI, total cholesterol, and systolic blood pressure compared with men [22]. These risk factors lead to higher mortality risk in women than in men. In a secondary analysis of the ADVANCE (Action in Diabetes and Vascular Disease: Preterax and Diamicron modified release Controlled Evaluation) trial, smoking contributed to an increase in CVD mortality in both sexes to a comparable extent with the exception of some evidence of a greater risk in women for major coronary events [41•]. Smoking cessation reduced all-cause mortality risk in both sexes by $30 \%$. Several studies have indicated that not only might there be more harm at the same level of risk factors, but that women with diabetes receive insufficient medical care, leading to a worse CVD risk factor and management profile $[30 \bullet, 31 \bullet, 33 \bullet, 42,43]$. This would further contribute to CVD outcome differences. Several such sex differences in CVD risk factors have been

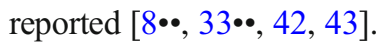


Sex differences in CVD risk factors reported have been mostly attributed to changes after menopause, including a higher risk of hypertension in women with diabetes, higher obesity prevalence aggravating diabetes, and contributing to additional risk for CVD through inflammatory processes, ectopic lipid accumulation, insulin resistance, and hypertension [33・•]. In women with type 2 diabetes, a more adverse cardiovascular risk profile has been described with higher metabolic syndrome prevalence, worse blood pressure control, and higher HbAlc. In women, hypertension, low physical activity, and high alcohol intake have been identified to be stronger predictors for acute myocardial infarction compared with men [44•]. A recently published meta-analysis of CVOTs found higher systolic blood pressure (mean difference $1.66 \mathrm{mmHg}$; $95 \%$ CI $0.90,2.41)$, LDL-C $(0.34 \mathrm{mmol} / \mathrm{L} ; 0.29,0.39)$, and HbAlc $(0.11 \% ; 0.09,0.14 ;[1.2 \mathrm{mmol} / \mathrm{mol} 1.0,1.5])$ in the baseline characteristics of women compared with men participating in these trials $[13 \cdot \bullet]$. A register study with more than 120,000 patients with T2DM found that those females aged $\geq$ 60 years, when compared with males, were more obese and more likely to have higher blood pressure and dyslipidemia rates but lower rates of lipid-lowering medication, worse glycemic control, and a higher risk of retinopathy [45]. In a further cross-sectional study investigating men and women with insulin-treated T2DM aged $>60$ years, women were reported to have higher levels of LDL-C and HDL-C, and higher systolic and diastolic blood pressures [46]. Furthermore, treatment goals for hypertension were achieved less often by women, despite comparable glycemia in both sexes. In a study investigating sex and gender aspects of cardiometabolic risk in T1DM, similar glycemic control but higher cholesterol in women, and higher triglyceride in men were reported [47]. Differences in cardiometabolic and CVD risk factors are already present in prediabetes and continue into diabetes: these sex differences might start early in life with higher female subclinical inflammation and coagulopathy evident from early adulthood [48]. More recent studies found that the highest relative risk sex difference for CVD events is before 60 years and highest in women with T2DM at ages 50-60 years [24•]. Differences in lipid storage and body composition driven by hormonal action seem to play an important role in this puzzle. Abdominal adiposity does increase in women with aging and a stronger obesity-diabetes risk association in women has been reported $[8 \bullet \bullet$. Usually, healthy women feature higher peripheral insulin sensitivity, which is reflected in lower absolute T2DM prevalence compared with men $[8 \cdot \bullet$. This means that in order to progress to T2DM, healthy women need to gain more weight than men. Indeed, women do have significantly higher BMI starting at younger ages when progressing to T2DM, particularly at younger ages [45]. Moreover, compared with men, women have a more distinct association between abdominal visceral adipose tissue and insulin resistance measured by HOMA IR and insulin secretion [49]. This has been corroborated by a further study demonstrating a higher risk for acute myocardial infarction in women with higher waist circumferences and waist-to-hip ratio compared with men [50•]. In comparison with nulliparous women, BMI was significantly higher in women with two or more children $\left(0.6 \mathrm{~kg} / \mathrm{m}^{2}\right.$ per child $)$ and significant worse cardiometabolic parameters with significantly lower HDL cholesterol were observed [51]. Postpartum weight changes were found to be relevant for cardiometabolic parameters with significantly higher blood pressure, HOMA-IR, LDL cholesterol, and apoB in those women who do not lose weight between 3 and 12 months after delivery [52].

\section{Sex Differences in Medication Effects, Side Effects, CV Risk Reduction Medications, and Adherence Among People with Type 2 Diabetes}

Among participants in the FIELD study, fenofibrate was associated with greater lipid-lowering effects in women and demonstrated a $30 \%$ reduction of CVD events in women and $13 \%$ in men [53]. Rosuvastatin was associated with greater atheroma volume regression (in percent) in women, but a comparable degree of total atheroma volume regression in both sexes after 24 months. Atorvastatin has been shown to have a similar degree of atheroma regression in men and women [30•]. A higher rate of side effects for statins was observed in women with increased liver enzymes and myalgia [54]. In male and female patients with type 2 diabetes, the benefits of the use of aspirin in secondary prevention of cardiovascular events were reported [30•]. In a recent metaanalysis with 12 RCTs including $>34,000$ individuals, aspirin use was found to be effective in primary prevention for MACE reduction of $11 \%$ (RR $0.89(0.83-0.95)$ ) with no sex interactions found [55]. During pregnancy, aspirin is recommended for preeclampsia prevention for high-risk women which includes T1DM and T2DM [56] but whether this intervention reduces later, CVD or CVD risk factors in women with diabetes is unknown.

Lower medication adherence in women than men has been reported. It has been postulated that this may be due to lower self-care activities in women as a result of higher depression rates, less problem-oriented actions, and fewer problem solving approaches which have been reported in several studies [30 $]$. Furthermore, higher rates of side effects leading to discontinuation of therapy were reported. A recently published meta analysis of CVOTs reported less use of beta-blockers (RR 0.93; 95\% CI 0.88, 0.97), aspirin $(0.82 ; 0.71,0.95)$, and statins $(0.90 ; 0.86,0.93)$ in women compared with men but comparable use of RAAS blockers (RR 1.00; 95\% CI 0.99, $1.05)[13 \cdot \bullet]$. Next to the reasons above, health care providers might underestimate CVD risk in women with diabetes, which 
results in less prescribing of CVD protective medication and thus suboptimal CVD risk factor management.

\section{Sex Differences in CVD Management Among People with Diabetes}

In women with T1DM, adherence to pharmacological intervention and cardio-protective measures was lower in women with guideline recommendations significantly less likely to be achieved by women for the target LDL-C levels, blood pressure, or aspirin prescription [47]. Intensive lifestyle interventions appear to be equally effective in men and women with diabetes in reducing weight, improving fitness, and maintaining healthy functioning. Similarly, prevention of progression from impaired glucose tolerance to diabetes is equally effective in men and women with a $37 \%$ lower progression rate to T2DM reported in a systematic review including 12 RCTs [57•]. Meanwhile, the DaQing study in China reported lower CVD mortality in female than male participants with prediabetes in the 23-year follow-up after lifestyle intervention [58]. These results demonstrate the effectiveness of intensive lifestyle modification in women with prediabetes and suggest that these approaches might also help to reduce CVD mortality in women with diabetes. However, this strategy needs to be verified in other cohorts. Sociocultural aspects cannot be translated easily to other cohorts and there was a substantial difference in smoking behavior - a serious CVD risk factor-between men and women within the DaQing study.

\section{Other Psychological and Socioeconomic Factors that Might Contribute to Increased CVD Risk Among Women}

Worse metabolic control among women with diabetes is associated with lower quality of life, higher external dependency, insomnia, reduced sexual desire, and lower satisfaction with social support [30•]. Similarly higher diabetes risk among women has been associated with socioeconomic status including low education levels, low socioeconomic status in childhood, and thereafter, high job strain with low decision latitude, shift work with night shifts causing sleep deprivation [8••]. In an Austrian health survey including $>13,500$ participants, only women showed an inverse association between educational level and diabetes and hypertension, with the highest risk at the lowest educational level [59]. In both sexes, overweight/obesity was higher with lower educational levels. A higher risk of T2DM was shown in Sweden with increasing age and among those of lower socioeconomic status which was accentuated in migrant women [60].
In a meta-analysis of sleep duration in the general population including $>3,500,000$ participants globally, a U-shaped risk pattern was found for (i) all-cause mortality (RR 1.06 (95\% CI, 1.04-1.07) per 1-h reduction and RR 1.13 (95\% CI, 1.11-1.15) per 1-h increment) and (ii) for cardiovascular mortality (RR 1.06 (95\% CI, 1.03-1.08) per 1 -h reduction and 1.12 (95\% CI, 1.081.16) per 1 -h increment). There were no sex differences and the lowest risk at around a sleep duration of $7 \mathrm{~h}$ a day [61]. Again, in the general population, the Nurses' Health Study revealed that a sleep duration of less than $5 \mathrm{~h}$ was closely linked with a higher incidence of hypertension in younger women [62•]. Findings of a large study including more than 700,000 people investigating the relationship of sleep duration and hypertension across age and sex found that both sexes are prone to a higher risk of hypertension with short and long sleeping durations as age increases [63]. The associations between short sleep duration were stronger among younger adults and women.

\section{Women with Gestational Diabetes: a Special Female Population with High Risk}

Women with prior GDM are among those with the highest risk for progression to T2DM and the metabolic syndrome [31•]. Recently, a Canadian systematic review with pooled analysis including more than 5,390,000 women and a total of 101,000 cardiovascular events reported an increased risk for CVD (RR 1.98, 95\% CI 1.57-2.50) up to 10 years postpartum for women with prior GDM and a subsequent diagnosis of T2DM [14••]. Interestingly, this risk was also increased in women with a history of GDM but no progression to T2DM with an RR of 1.56 (1.04-2.32). Women with GDM often suffer from hypertensive disorders in pregnancy. A twofold higher risk for CVD compared with women with normotensive pregnancies was reported in women with hypertensive disorders [64]. Both GDM and hypertensive disorders in pregnancy might add up to further increased CVD risk in later life. Intensive lifestyle intervention as reported in the "Gestational Diabetes' Effects on Moms "(GEM) study was able to increase vigorous physical activity and decrease weight gain retention by $-0.64 \mathrm{~kg}(95 \% \mathrm{CI}-$ $1.13,-0.14)$ at 6 months postpartum in women with a history of GDM compared with usual care [65]. Women with PCOS are also at an increased risk for the development of both T2DM and CVD risk factors with their higher rates of overweight/obesity including central obesity and dyslipidemia. Although predicted, a higher risk for CVD mortality has not so far been reported due to the paucity of population-based long-term studies [66, 67]. 


\section{What Can Be Done to Reduce CVD Mortality Rates Among Women?}

It may be possible to reduce CVD mortality rates among women through a range of strategies (Fig. 1). Firstly, increased awareness of the issue, including sex- and genderspecific guidelines, may be a starting point, and are consistent with the move to more personalized care. Such actions have already commenced in the treatment of those with prevalent and incident CVD [68]. This has decreased sex disparities although they are still existing and keep the "Yentl syndrome" alive (the Yentl Syndrome is the different course of action that myocardial infarctions usually follow for women than for men) $[69 \bullet \bullet, 70]$. Further work includes further implementation of standardized protocols, the use of m- and e-health tools to increase awareness, and the use of artificial intelligence technology.

Improved management of CVD risk factors among women is also required, again through awareness, education, guidelines, and protocols, and also possibly through earlier management of hypertension, dyslipidemia, excess weight, and hyperglycemia. Whether metabolic targets need to be lower for women is unclear [71].

Further work is needed to address gender differences in adherence which also appear to be central in the progression to T2DM and CVD. For example, depression is more prevalent in women with prediabetes and diabetes and might explain some of the differences in treatment adherence [48]. Depression can be aggravated with worse glycemic control, potentially further contributing to reduced self-management and thus increasing mortality risk. Improved depression management in women with diabetes and prediabetes may therefore address some of the CVD protection loss.

Evidence for additional and more intensive (some would say aggressive) treatment to improve CVD outcomes still needs scientific proof, but several strategies have been shown to be effective for reducing CVD events before diabetes has developed including lifestyle interventions in women with prediabetes, with a history of GDM, hypertensive disorders in pregnancy, and PCOS [57•, 72, 73••]. Further evidence is needed to open further opportunities for the prevention of CVD events among women at risk of diabetes.

Further research into sex and gender differences is important to understand the biological basis of these sex disparities further, how to improve clinical, more personalized care, alongside studies on how to increase awareness and understanding of women, their partners, and health care professionals.

The associations of sex hormones and glucose metabolism as well as other hormones such as GLP1 need further investigation, as they seem to have an important role in energy regulation and are involved in beta cell protection [36]. New findings and further research might pave the way for sex-specific targeted therapeutic modalities, as for example selective estrogen receptor modulators in postmenopausal women, GLP-1estrogen conjugate pathways or selective ER $\alpha$ activation [36].

\section{Conclusion}

We conclude that women need additional and more intensive cardiovascular risk reduction strategies beyond those currently available to men with diabetes. Women with diabetes have a higher relative CVD mortality risk, due to the loss of their "natural" protection with progression to diabetes. Evidence from recent studies demonstrates that this is especially true for younger age groups where sex disparities are more prevalent and for women with prior GDM. Whether more aggressive strategies are required such as lower metabolic targets, greater polypharmacy earlier in the disease course is unknown and warrant urgent trials.

Interventions have commenced [60], including adaptations to guidelines and protocols, but further work is needed to address gender differences in adherence through improved management of co-morbidities, e.g., depression and improved CVD risk factor management. A key area to be addressed urgently is the follow-up of women with prior GDM or hypertensive disorders in pregnancy to systematically reduce progression to T2DM and CVD through evidence-based programs and services.

Funding Open access funding provided by Medical University of Vienna. Alexandra Kautzky-Willer received funding from the WWTF (Vienna Science and Technology Fund -MA16-045). Alexandra Kautzky-Willer and Jürgen Harreiter are participating in the project Gender Outcomes INternational Group: to Further Well-being Development (GOING-FWD), which receives funding from FWF (Austrian Science Fund) and GENDER-NET Plus ERA-NET cofund.

\section{Compliance with Ethical Standards}

Conflict of Interest The authors declare that they have no conflict of interest.

Human and Animal Rights and Informed Consent This article does not contain any studies with human or animal subjects performed by any of the authors.

Open Access This article is licensed under a Creative Commons Attribution 4.0 International License, which permits use, sharing, adaptation, distribution and reproduction in any medium or format, as long as you give appropriate credit to the original author(s) and the source, provide a link to the Creative Commons licence, and indicate if changes were made. The images or other third party material in this article are included in the article's Creative Commons licence, unless indicated otherwise in a credit line to the material. If material is not included in the article's Creative Commons licence and your intended use is not permitted by statutory regulation or exceeds the permitted use, you will need to obtain permission directly from the copyright holder. To view a copy of this licence, visit http://creativecommons.org/licenses/by/4.0/. 


\section{References}

Papers of particular interest, published recently, have been highlighted as:

- Of importance

- Of major importance

1. WHO. World health statistics 2018: monitoring health for the Sustainable Development Goals. 2019. Available from: www. who.int/gho/publications/world_health_statistics/2018/en. Accessed 9 May 2020.

2. Sundberg L, Agahi N, Fritzell J, Fors S. Why is the gender gap in life expectancy decreasing? The impact of age- and cause-specific mortality in Sweden 1997-2014. Int J Public Health. 2018;63(6): 673-81.

3. Luy M, Wegner-Siegmundt C. The impact of smoking on gender differences in life expectancy: more heterogeneous than often stated. Eur J Pub Health. 2015;25(4):706-10.

4. Luy M, Gast K. Do women live longer or do men die earlier? Reflections on the causes of sex differences in life expectancy. Gerontology. 2014;60(2):143-53.

5. Van Oyen H, Nusselder W, Jagger C, et al. Gender differences in healthy life years within the EU: an exploration of the "healthsurvival" paradox. Int J Public Health. 2013;58(1):143-55.

6. EUROSTAT. Cardiovascular disease statistics 2019 Available from: https:/ec.europa.eu/eurostat/statistics-explained/pdfscache/ 37359.pdf. Accessed 30 Jan 2020.

7. International Diabetes Federation. IDF Diabetes Atlas 9th Edition 2019 Available from: https://www.diabetesatlas.org/en/. Accessed 30 Jan 2020.

8.• Kautzky-Willer A, Harreiter J, Pacini G. Sex and gender differences in risk, pathophysiology and complications of type 2 diabetes mellitus. Endocr Rev. 2016;37(3):278-316 Comprehensive review summarizing sex and gender differences in T2DM.

9. Garcia MJ, McNamara PM, Gordon T, Kannel WB. Morbidity and mortality in diabetics in the Framingham population. Sixteen year follow-up study. Diabetes. 1974;23(2):105-11.

10. Peters SA, Huxley RR, Woodward M. Diabetes as risk factor for incident coronary heart disease in women compared with men: a systematic review and meta-analysis of 64 cohorts including 858 , 507 individuals and 28,203 coronary events. Diabetologia. 2014;57(8):1542-51 Higher CHD risk in women.

11. Kalyani RR, Lazo M, Ouyang P, Turkbey E, Chevalier K, Brancati $\mathrm{F}$, et al. Sex differences in diabetes and risk of incident coronary artery disease in healthy young and middle-aged adults. Diabetes Care. 2014;37(3):830-8.

12. Huxley RR, Peters SA, Mishra GD, Woodward M. Risk of allcause mortality and vascular events in women versus men with type 1 diabetes: a systematic review and meta-analysis. Lancet Diabetes Endocrinol. 2015;3(3):198-206 Increased risk for all-cause mortality and CVD mortality in women with T1DM.

13.• Clemens KK, Woodward M, Neal B, Zinman B. Sex Disparities in cardiovascular outcome trials of populations with diabetes: a systematic review and meta-analysis. Diabetes Care. 2020;43(5): 1157-63 Meta-analysis of CVOTs with higher risk for stroke, heart failurs and CKD in women.

14.• Kramer CK, Campbell S, Retnakaran R. Gestational diabetes and the risk of cardiovascular disease in women: a systematic review and meta-analysis. Diabetologia. 2019;62(6):905-14 Increased cardiovascular risk after GDM.

15. Peters SA, Huxley RR, Sattar N, Woodward M. Sex differences in the excess risk of cardiovascular diseases associated with type 2 diabetes: potential explanations and clinical implications. Curr Cardiovasc Risk Rep. 2015;9(7):36.
16. Ljungman C, Kahan T, Schioler L, et al. Gender differences in antihypertensive drug treatment: results from the Swedish primary care cardiovascular database (SPCCD). J Am Soc Hypertens. 2014;8(12):882-90.

17. Population Reference Bureau. Average life expectancy at birth in 2019, by continent and gender (in years). 2019. https://www.prb. org/international/. Accessed 2 Feb 2020.

18. Luy M. Causes of male excess mortality: insights from cloistered populations. Popul Dev Rev. 2003;29(4):647-76.

19. Xu G, You D, Wong L, Duan D, Kong F, Zhang X, et al. Risk of all-cause and CHD mortality in women versus men with type 2 diabetes: a systematic review and meta-analysis. Eur J Endocrinol. 2019;180(4):243-55.

20. Wang Y, O'Neil A, Jiao Y, et al. Sex differences in the association between diabetes and risk of cardiovascular disease, cancer, and allcause and cause-specific mortality: a systematic review and metaanalysis of 5,162,654 participants. BMC Med. 2019;17(1):136.

21. Huxley R, Barzi F, Woodward M. Excess risk of fatal coronary heart disease associated with diabetes in men and women: metaanalysis of 37 prospective cohort studies. BMJ. 2006;332(7533): 73-8 Higher risk of fatal CHD in women with diabetes vs. men.

22. Prospective Studies C. Asia Pacific Cohort Studies C. Sex-specific relevance of diabetes to occlusive vascular and other mortality: a collaborative meta-analysis of individual data from 980793 adults from 68 prospective studies. Lancet Diabetes Endocrinol. 2018;6(7):538-46.

23. Wright AK, Kontopantelis E, Emsley R, Buchan I, Mamas MA, Sattar N, et al. Cardiovascular risk and risk factor management in type 2 diabetes mellitus. Circulation. 2019;139(24):2742-53.

24. Malmborg M, MDS S, Norgaard CH, et al. Does type 2 diabetes confer higher relative rates of cardiovascular events in women compared with men? Eur Heart J. 2019;41(13):1346-53 Higher absolute risk of MACE-HF in men, but in relative terms higher women to men ratio.

25. Ohkuma T, Komorita Y, SAE P, Woodward M. Diabetes as a risk factor for heart failure in women and men: a systematic review and meta-analysis of 47 cohorts including 12 million individuals. Diabetologia. 2019;62(9):1550-60 Women vs men with diabetes have increased risk of heart failure.

26. Kannel WB, Hjortland M, Castelli WP. Role of diabetes in congestive heart failure: the Framingham study. Am J Cardiol. 1974;34(1): 29-34.

27. Hu G, Jousilahti P, Qiao Q, Katoh S, Tuomilehto J. Sex differences in cardiovascular and total mortality among diabetic and nondiabetic individuals with or without history of myocardial infarction. Diabetologia. 2005;48(5):856-61.

28. Dantas AP, Fortes ZB, de Carvalho MH. Vascular disease in diabetic women: why do they miss the female protection? Exp Diabetes Res. 2012;2012:570598.

29. Booth GL, Kapral MK, Fung K, Tu JV. Relation between age and cardiovascular disease in men and women with diabetes compared with non-diabetic people: a population-based retrospective cohort study. Lancet. 2006;368(9529):29-36.

30. Kautzky-Willer A, Harreiter J. Sex and gender differences in therapy of type 2 diabetes. Diabetes Res Clin Pract. 2017;131:230-41 Comprehensive review summarizing sex and gender differences in T2DM management.

31. Harreiter J, Kautzky-Willer A. Sex and gender differences in prevention of type 2 diabetes. Front Endocrinol. 2018;9:220 Comprehensive review summarizing sex and gender differences in prevention of T2DM.

32. Bokslag A, Teunissen PW, Franssen C, et al. Effect of early-onset preeclampsia on cardiovascular risk in the fifth decade of life. Am J Obstet Gynecol. 2017;216(5):523 e1-7.

33.• Regensteiner JG, Golden S, Huebschmann AG, et al. Sex differences in the cardiovascular consequences of diabetes mellitus: a 
scientific statement from the American Heart Association. Circulation. 2015;132(25):2424-47 AHA statement highlighting the need for more attention regarding sex and gender differences in CVD and Diabetes Mellitus.

34. Lovejoy JC, Sainsbury A. Stock Conference Working G. Sex differences in obesity and the regulation of energy homeostasis. Obes Rev. 2009;10(2):154-67.

35.• Mauvais-Jarvis F, Manson JE, Stevenson JC, Fonseca VA. Menopausal hormone therapy and type 2 diabetes prevention: evidence, mechanisms, and clinical implications. Endocr Rev. 2017;38(3):173-88 Comprehensive report about (patho-)physiology of female sex hormones in T2DM and T2DM prevention.

36. Tramunt B, Smati S, Grandgeorge N, Lenfant F, Arnal JF, Montagner A, et al. Sex differences in metabolic regulation and diabetes susceptibility. Diabetologia. 2020;63(3):453-61.

37.• Ding EL, Song Y, Malik VS, Liu S. Sex differences of endogenous sex hormones and risk of type 2 diabetes: a systematic review and meta-analysis. JAMA. 2006;295(11):1288-99 Male and female sex hormones have different effects on diabetes risk in the other sex.

38. Anagnostis P, Tarlatzis BC, Kauffman RP. Polycystic ovarian syndrome (PCOS): long-term metabolic consequences. Metabolism. 2018;86:33-43.

39. Nestler JE, Jakubowicz DJ, de Vargas AF, et al. Insulin stimulates testosterone biosynthesis by human thecal cells from women with polycystic ovary syndrome by activating its own receptor and using inositolglycan mediators as the signal transduction system. J Clin Endocrinol Metab. 1998;83(6):2001-5.

40. Harreiter J, Kautzky-Willer A. Gender obesity report-influence of obesity on reproduction and pregnancy. Wien Med Wochenschr. 2016;166(3-4):129-38

41. Blomster JI, Woodward M, Zoungas S, et al. The harms of smoking and benefits of smoking cessation in women compared with men with type 2 diabetes: an observational analysis of the ADVANCE (Action in Diabetes and Vascular Disease: Preterax and Diamicron modified release Controlled Evaluation) trial. BMJ Open. 2016;6(1):e009668 In men and women, smoking contributes to an increase in CVD mortality to a comparable extent.

42. de Ritter R, de Jong M, Vos RC, van der Kallen CJH, Sep SJS, Woodward M, et al. Sex differences in the risk of vascular disease associated with diabetes. Biol Sex Differ. 2020;11(1):1.

43. Huebschmann AG, Huxley RR, Kohrt WM, Zeitler P, Regensteiner JG, Reusch JEB. Sex differences in the burden of type 2 diabetes and cardiovascular risk across the life course. Diabetologia. 2019;62(10):1761-72.

44. Anand SS, Islam S, Rosengren A, et al. Risk factors for myocardial infarction in women and men: insights from the INTERHEART study. Eur Heart J. 2008;29(7):932-40 Women with diabetes have a more adverse cardiovascular risk profile.

45. Awa WL, Fach E, Krakow D, Welp R, Kunder J, Voll A, et al. Type 2 diabetes from pediatric to geriatric age: analysis of gender and obesity among 120,183 patients from the German/Austrian DPV database. Eur J Endocrinol. 2012;167(2):245-54.

46. Gobl CS, Brannath W, Bozkurt L, et al. Sex-specific differences in glycemic control and cardiovascular risk factors in older patients with insulin-treated type 2 diabetes mellitus. Gend Med. 2010;7(6): 593-9.

47. Kautzky-Willer A, Stich K, Hintersteiner J, Kautzky A, Kamyar M, Saukel J, et al. Sex-specific-differences in cardiometabolic risk in type 1 diabetes: a cross-sectional study. Cardiovasc Diabetol. 2013;12:78.

48. Breyer MK, Ofenheimer A, Altziebler J, et al. Marked differences in prediabetes and diabetes associated comorbidities between men and women - epidemiological results from a general population- based cohort aged 6-80 years - the LEAD (Lung, hEart, sociAl, boDy) study. Eur J Clin Investig. 2020;50(3):e13207.

49. de Mutsert R, Gast K, Widya R, de Koning E, Jazet I, Lamb H, et al. Associations of abdominal subcutaneous and visceral fat with insulin resistance and secretion differ between men and women: the Netherlands epidemiology of obesity study. Metab Syndr Relat Disord. 2018;16(1):54-63.

50. SAE P, Bots SH, Woodward M. Sex differences in the association between measures of general and central adiposity and the risk of myocardial infarction: results from the UK Biobank. J Am Heart Assoc. 2018;7(5):e008507 Higher risk for acute myocardial infarction in women with higher waist circumferences and waistto-hip ratio compared with men.

51. Zoet GA, Paauw ND, Groenhof K, Franx A, Gansevoort RT, Groen $\mathrm{H}$, et al. Association between parity and persistent weight gain at age 40-60 years: a longitudinal prospective cohort study. BMJ Open. 2019;9(5):e024279.

52. Kew S, Ye C, Hanley AJ, Connelly PW, Sermer M, Zinman B, et al. Cardiometabolic implications of postpartum weight changes in the first year after delivery. Diabetes Care. 2014;37(7):19982006.

53. d'Emden MC, Jenkins AJ, Li L, et al. Favourable effects of fenofibrate on lipids and cardiovascular disease in women with type 2 diabetes: results from the Fenofibrate Intervention and Event Lowering in Diabetes (FIELD) study. Diabetologia. 2014;57(11): 2296-303.

54. Goldstein LB, Amarenco P, Lamonte M, Gilbert S, Messig M, Callahan A, et al. Relative effects of statin therapy on stroke and cardiovascular events in men and women: secondary analysis of the Stroke Prevention by Aggressive Reduction in Cholesterol Levels (SPARCL) Study. Stroke. 2008;39(9):2444-8.

55. Seidu S, Kunutsor SK, Sesso HD, Gaziano JM, Buring JE, Roncaglioni $\mathrm{MC}$, et al. Aspirin has potential benefits for primary prevention of cardiovascular outcomes in diabetes: updated literature-based and individual participant data meta-analyses of randomized controlled trials. Cardiovasc Diabetol. 2019;18(1):70.

56. ACOG Committee Opinion No. 743: low-dose aspirin use during pregnancy. Obstet Gynecol. 2018;132(1):e44-52.

57. Glechner A, Harreiter J, Gartlehner G, et al. Sex-specific differences in diabetes prevention: a systematic review and meta-analysis. Diabetologia. 2015;58(2):242-54 Comparable effects of lifestyle intervention on diabetes prevention in men and women.

58. Li G, Zhang P, Wang J, An Y, Gong Q, Gregg EW, et al. Cardiovascular mortality, all-cause mortality, and diabetes incidence after lifestyle intervention for people with impaired glucose tolerance in the Da Qing Diabetes Prevention Study: a 23-year follow-up study. Lancet Diabetes Endocrinol. 2014;2(6):474-80.

59. Kautzky-Willer A, Dorner T, Jensby A, Rieder A. Women show a closer association between educational level and hypertension or diabetes mellitus than males: a secondary analysis from the Austrian HIS. BMC Public Health. 2012;12:392.

60. Wemrell M, Bennet L, Merlo J. Understanding the complexity of socioeconomic disparities in type 2 diabetes risk: a study of 4.3 million people in Sweden. BMJ Open Diabetes Res Care. 2019;7(1):e000749.

61. Yin J, Jin X, Shan Z, et al. Relationship of sleep duration with allcause mortality and cardiovascular events: a systematic review and dose-response meta-analysis of prospective cohort studies. J Am Heart Assoc. 2017;6(9):e005947.

62. Gangwisch JE, Feskanich D, Malaspina D, Shen S, Forman JP. Sleep duration and risk for hypertension in women: results from the Nurses' Health Study. Am J Hypertens. 2013;26(7):903-11 Ushaped association of sleep duration with CVD and all cause mortality.

63. Grandner M, Mullington JM, Hashmi SD, Redeker NS, Watson NF, Morgenthaler TI. Sleep duration and hypertension: analysis 
of $>700,000$ adults by age and sex. J Clin Sleep Med. 2018;14(6): 1031-9.

64. Benschop L, Duvekot JJ. Roeters van Lennep JE. Future risk of cardiovascular disease risk factors and events in women after a hypertensive disorder of pregnancy. Heart. 2019;105(16):1273-8.

65. Ferrara A, Hedderson MM, Brown SD, Albright CL, Ehrlich SF, Tsai AL, et al. The comparative effectiveness of diabetes prevention strategies to reduce postpartum weight retention in women with gestational diabetes mellitus: the Gestational Diabetes' Effects on Moms (GEM) Cluster Randomized Controlled Trial. Diabetes Care. 2016;39(1):65-74.

66. Jacewicz-Swiecka M, Kowalska I. Polycystic ovary syndrome and the risk of cardiometabolic complications in longitudinal studies. Diabetes Metab Res Rev. 2018;34(8):e3054.

67. Kakoly NS, Moran LJ, Teede HJ, Joham AE. Cardiometabolic risks in PCOS: a review of the current state of knowledge. Expert Rev Endocrinol Metab. 2019;14(1):23-33.

68. Benjamin EJ, Muntner P, Alonso A, Bittencourt MS, Callaway CW, Carson AP, et al. Heart disease and stroke statistics-2019 update: a report from the American Heart Association. Circulation. 2019;139(10):e56-e528.
69.•• Healy B. The Yentl syndrome. N Engl J Med. 1991;325(4):274-6 Women need to act like men to receive the same treatment

70. Merz CN. The Yentl Syndrome is alive and well. Eur Heart J. 2011;32(11):1313-5.

71. Simmons D. Excess deaths in women with type 1 diabetes: time to act. Lancet Diabetes Endocrinol. 2015;3(3):164-5.

72. Woodward A, Broom D, Harrop D, Lahart I, Carter A, Dalton C, et al. The effects of physical exercise on cardiometabolic outcomes in women with polycystic ovary syndrome not taking the oral contraceptive pill: a systematic review and meta-analysis. Syst Rev. 2019;18(2):597-612.

73.• Aroda VR, Christophi CA, Edelstein SL, et al. The effect of lifestyle intervention and metformin on preventing or delaying diabetes among women with and without gestational diabetes: the Diabetes Prevention Program outcomes study 10-year follow-up. J Clin Endocrinol Metab. 2015;100(4):1646-53 prevention of T2DM after GDM.

Publisher's Note Springer Nature remains neutral with regard to jurisdictional claims in published maps and institutional affiliations. 